\title{
Le génome d'une archaéobactérie entièrement séquencé: la génomique comparative peut commencer
}

L'ère de la véritable comparaison entre les génomes ou "génomique comparative " vient de s'ouvrir avec la publication de la séquence complète du génome de l'archaéobactérie méthanogène Methanococcus jannaschii [1] par un groupe américain du «TIGR" (Institut for Genomic Research) dirigé par Craig Venter. Ce même groupe avait déjà rapporté la séquence complète de deux procaryotes (Haemophilus influenzae $[\mathrm{m} / \mathrm{s}$ $n^{\circ} 12$, vol. 11, p. 1761] et Mycoplasma genitalium), et l'annonce vient d'être faite de la réalisation de la séquence complète des 16 chromosomes de l'eucaryote Saccharomyces cerevisiae [2]. On commence, en outre, à avoir de vastes banques de données pour Homo sapiens et Caenorhabditis elegans. Il manquait donc une séquence du groupe des archaéobactéries qui constitue, avec les procaryotes et les eucaryotes, les trois règnes primaires du monde vivant [3], pour pouvoir utiliser une approche évolutionniste comparative entre leurs représentants.

Cette approche devrait permettre de comprendre les bases moléculaires de l'origine et de la diversification de la vie cellulaire. $M$. jannaschii a été isolé en 1982 à partir de sédiments isolés à $3000 \mathrm{~m}$ de profondeur dans l'océan Pacifique. C'est un anaérobie strict qui pousse sous une pression de 200 atmosphères, à une température de 48 à $94^{\circ} \mathrm{C}$ et qui, de plus, comme son nom l'indique, produit du méthane.

Pour le séquençage, une approche de séquençage de tout le génome au hasard a été choisie, comme pour Haemophilus influenzae $\left(\mathrm{m} / \mathrm{s} n^{\circ} 12\right.$, vol. 11, p. 1761). Cette méthode, controversée au début mais maintenant largement acceptée, court-circuite l'étape classique de la construction de la carte faite de petits clones d'ADN chevauchants. L'ADN est séquencé avant que l'on ne connaisse l'arrangement des clones et ce sont des ordinateurs qui assemblent l'ensemble de la séquence.

Le groupe de Craig Venter a d'abord réalisé deux banques d'ADN, l'une contenant de grands inserts dans des phages, l'autre des petits inserts dans des plasmides. Le génome de la bactérie a été divisé en 36000 petits fragments de 2,5 kb chacun, puis la séquence des deux extrémités de 17000 clones a été réalisée. Un programme informatique a alors recherché les séquences chevauchantes de plus de 50 pb et a assemblé l'ensemble des séquences en quatorze groupes ou contigs. Il restait alors à réunir ces quatorze groupes. C'est là qu'intervient la deuxième banque de 400 à 500 clones contenant de grands fragments de $15 \mathrm{~kb}$ chacun. Les extrémités de ces clones ont été séquencées, ce qui a permis de créer un réseau dans lequel ont pu être placées les séquences des quatorze groupes précédents et donc de combler les trous. Il aura fallu seulement un an au groupe du «TIGR" pour réaliser ce travail.

Le génome de $M$. jannaschii se compose de trois éléments physiques distincts: (1) un grand chromosome circulaire de 1664976 pb contenant 1682 régions codantes prédites avec un contenu G + C de 31,4\%; (2) un grand élément circulaire extrachromosomique de $58407 \mathrm{pb}$ contenant
44 régions codantes prédites avec un contenu en $\mathrm{G}+\mathrm{C}$ de $28,2 \%$; (3) un petit élément extrachromosomique circulaire de $16550 \mathrm{pb}$ qui contient 12 régions codantes prédites avec un contenu en $\mathrm{G}+\mathrm{C}$ de $28,8 \%$. La première surprise lorsque l'on a comparé les séquences de $M$. jannaschii avec celles déjà disponibles est que $38 \%$ seulement des régions codantes prédites ont des similitudes avec un gène dont on connaît la fonction cellulaire et $6 \%$ des similitudes avec d'hypothétiques protéines. Ces $44 \%$ de similitude avec des gènes déjà rapportés contrastent avec les $78 \%$ de similitude trouvés pour les génomes des procaryotes $H$. influenza et M.jannaschii. La deuxième surprise est qu'il y a chez M. genitalium des gènes ressemblant totalement à ceux des procaryotes et d'autres qui ressemblent à ceux des eucaryotes [4]. La majorité des gènes impliqués dans la production d'énergie, la division cellulaire et le métabolisme chez M.jannaschii ont une similitude avec ceux trouvés chez les bactéries alors que la plupart des gènes impliqués dans la transcription, traduction $\left(\mathrm{m} / \mathrm{s} n^{\circ} 12\right.$, vol. 12, p. 1441) et réplication sont similaires à ceux présents chez les eucaryotes.

Le génome de M.jannaschii est aussi surprenant par ce qu'il n'a pas. Par exemple, il ne possède apparemment qu'une ADN polymérase pour se répliquer alors qu'il en faut trois chez Escherichia coli. De même, plusieurs enzymes chargeant les ARN de transfert semblent manquer chez cette archaéobactérie. Enfin, les auteurs ont été surpris par la présence de nombreuses intéines (18 en 
tout). Les intéines, appelées ainsi en analogie avec les introns, sont des portions de séquences de peptides capables d'autoépissage qui ont une activité d'endonucléase spécifique de site [5]. Les séquences restant après l'excision des intéines sont appelées extéines en analogie avec les exons. Les extéines sont liées entre elles après l'excision du ou des intéines pour former la protéine active. La signification biologique et le rôle des intéines ne sont pas clairement élucidés mais le fait que quatorze gènes dans le génome de $M$. jannaschii contiennent 18 intéines alors que jusqu'à ce jour seulement une dizaine de gènes contenant des intéines a été rapportée est assez remarquable.
Deux autres archaéobactéries sont en cours de séquençage: Sulfolobus solfataricus P2 [6] et Methanabacterium thermoautotrophicum [4]. Ces séquences ainsi que celles de formes plus primitives de bactéries et d'eucaryotes devraient permettre au scientifique de remonter à la racine de l'arbre de la vie. "Quand nous aurons assez de séquences, nous mettrons en évidence les choses qui sont communes à toutes les formes de la vie et montrerons comment les autres divergent par rapport à ce noyau ", confiait Carl Woese, microbiologiste et co-auteur du travail [4].

E.D.
1. Bult CJ, White O, Olsen GJ, Zhou L, Fleischamnn RD, et al. (40 auteurs). Complete genome sequence of the methanogenic archaeon, Methanococus jannaschii. Science 1996; 273: 105873.

2. Williams $\mathbf{N}$. Yeast genome sequence ferments new research. Science 1996; 272 : 481.

3. Denamur E, Picard B. De la genétique des populations bactériennes à l'épidémiologie des maladies infectieuses. Med Sci 1995; 11: 1399-406. 4. Morell V. Life's last domain. Science 1996; 273 : 1043-5.

5. Cooper AA, Stevens TH. Protein splicing: selfsplicing of genetically mobile elements at the protein level. Trends Biochem Sci 1995; 20 : 351-6.

6. Sensen CW, Klenk HP, Singh RK, Allard G, Chan CCY, Liu QY, Penny SL, Young F, Schenk ME, Gaasterland T, Doolittle WF, Ragan MA, Charlebois RL. Organizational characteristics and inforrnation content of an archaeal genome: $156 \mathrm{~kb}$ of sequence from Sulfolobus solfataricus $P 2$. Mol Microbiol 1996; 22: 175-91.

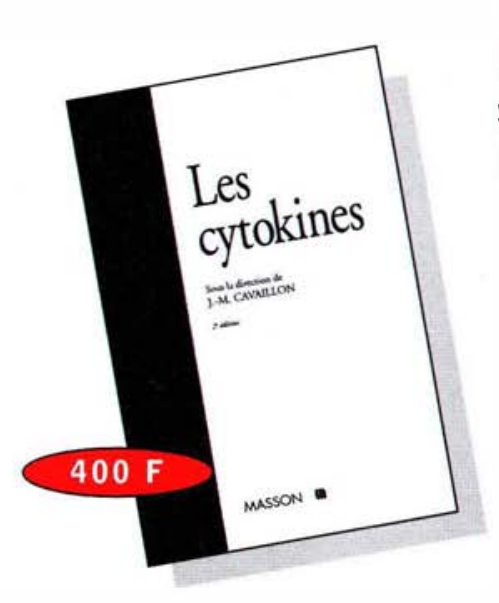

\section{Les cytokines}

nouvelle édition

\section{SOUS LA DiRECTION DE J.-M. CavaiLlon}

Préface de A. Capron

Toutes les cytokines sont traitées individuellement, et pour chacune, l'auteur présente un rappel historique de sa découverte, ses caractéristiques biochimiques et génétiques, la nature de son récepteur, ses sources, ses cibles privilégiées, ses activités biologiques, ses inhibiteurs, et sa possible implication dans la pathologie. D'autres chapitres présentent la participation des cytokines au cours de la réponse immunitaire, des mécanismes immunitaires et lors des troubles de l'hématopoï̀se. Pour tenir compte des progrès accomplis, de nouveaux thèmes ont été inclus ou développés (récepteurs et signaux de transduction, cytokines et transplantation, cytokines et réponse allergique, cytokines et reproduction).

1996, 2e édition, 624 pages, $16 \times 24$, broché

\section{MASSON U Ouvrage en vente en librairie ou à Saint-Germain Services}

A COMPLETER Et A RETOURner A SAINT-Germain SERVICES - BP 22 - 41354 Vineuil CEDEX

Je désire commander:

....... ex. de Les cytokines

par J.-M. Cavaillon à 400 F* (ISBN 2-225-85039-9)

Ci-joint mon chèque de .......... F libellé à l'ordre de Masson

*Prix public TTC au 01.05 .96
Nom .

Adresse

Code Postal

Frais d'envoi : pour 1 vol. $20 \mathrm{~F}$ (étranger : $30 \mathrm{~F}$ ), pour chaque volume supplémentaire $10 \mathrm{~F}$.

Franco de port pour toute commande supérieure à $1000 \mathrm{~F}$. Bon de commande 